\title{
Embedded Atom Method for Theoretical Strength and Stability of Some fcc Metals
}

\author{
Y. ÖZTEKIN C̣IfTCI* AND K. C̣OLAKOĞLU \\ Gazi University, Faculty of Art and Science, Ankara, Türkiye
}

(Received June 11, 2001)

\begin{abstract}
The structural phase transformation and theoretical strength of fcc metals $\mathrm{Ni}, \mathrm{Cu}, \mathrm{Ag}, \mathrm{Al}, \mathrm{Au}$, and $\mathrm{Pt}$ under [100] uniaxial loading are studied by using analytical embedded-atom-potential method. In the present calculations the stress-free bcc phase is found unstable and fcc phase is found to be stable. The obtained energy differences of $f_{c c}-b c c$ phases are comparable with those found by the first-principles calculations and experiments for all metals considered. The present pair potential in the embedded-atom method is used for the first time for this purpose. Theoretical lattice parameters, volumes, and energies of the bcc and fcc structures for each metal at zero pressure are calculated and compared with the available experimental values. Third-order elastic constants and pressure-volume curves for studied metals are also investigated and found generally good agreement with experiments.
\end{abstract}

PACS numbers: $64.60 .-\mathrm{i}, 62.20 .-\mathrm{x}$

\section{Introduction}

As is well known, the theoretical strength of a perfect crystal is defined as the strength at which a perfect lattice becomes mechanically unstable [1]. Furthermore, the theoretical strength represents an upper bound to the actual strength of solids, so its calculation is essential. Many works appear in the literature in the past three decades for analysis, based on the crystal lattice theory, the theoretical strength of cubic crystals in the presence of applied forces and deformations (see, particularly, Milstein's papers and the citations therein [2-23]).

*corresponding author; e-mail: yciftci@quark.fef.gazi.edu.tr 
The role and importance of the "theoretical strength" occurring in the elastic deformations in solids are emphasized by several authors [20, 24], and are not repeated here.

Since many elements transform from one crystal structure to another as the temperature is varied or by elastic deformation, theoretical structural phase transformations are, also, studied on these lines in the same period $[3,10,16,17,23$, 25-27]. A comprehensive description of the different path of deformations has been discussed by Milstein [8,18] and references therein in which uniaxial stress is applied to a tetragonal state along the [001] axis accompanied by zero stress in the (001) plane, and this path is the so-called uniaxial Bain path. The calculations on cubic crystals loaded uniaxially, generally, are treated by using the pairwise potential $[3,23,27,28]$, pseudopotential $[16,17,29,30]$, first-principle [31-33] and embedded-atom method $[18,25,34]$, references here are representative rather than exhaustive.

In this paper, we present a modified pair potential for analytical embedded-atom method (EAM) for investigating the theoretical strength and structural phase transformation, under [100] uniaxial loading for some fcc metals ( $\mathrm{Ni}, \mathrm{Cu}$, $\mathrm{Ag}, \mathrm{Al}, \mathrm{Au}, \mathrm{Pt}$ ) following the procedure given by Cai [25]. Third-order elastic constants (TOEC) and pressure-volume relations are also studied for the same metals.

\section{Model}

\subsection{Potential function and formulations}

According to the embedded-atom method, the cohesive energy of an assembly of $N$ atoms is given as [35]

$$
\begin{aligned}
& E_{\mathrm{t}}=\sum_{i} F_{i}\left(\rho_{i}\right)+\frac{1}{2} \sum_{i>j} \phi\left(r_{i j}\right), \\
& \rho_{i}=\sum f\left(r_{i j}\right),
\end{aligned}
$$

where $E_{\mathrm{t}}$ is the total internal energy, $\rho$ is the host electron density at atom $i$ due to all other atoms, $f\left(r_{i j}\right)$ is the electron density distribution function of an atom, $r_{i j}$ is the separation distance between atom $i$ and atom $j, F_{i}\left(\rho_{i}\right)$ is the embedding energy to embedded atom $i$ in an electron density $\rho_{i}$, and $\phi\left(r_{i j}\right)$ is the pairwise potential between atom $i$ and atom $j$. This model is widely used in the literature $[18,25,34,36-38]$ and references therein.

In this work, we propose a pair potential for the framework of EAM which is a modified form of the potential given by Cai [25]. The present form of the potential makes it more flexible due to the constants $m$ and $n$ in the multiplier forms. Such a factor included in the classical Morse function is treated by Verma and Rathore [39] to compute the phonon frequencies of Th by using the central pair potential 
TABLE I

Experimental data (with superscript letters) and calculated fitting parameters.

\begin{tabular}{c|c|c|c|c|c|c}
\hline \hline & $\mathrm{Ni}$ & $\mathrm{Cu}$ & $\mathrm{Al}$ & $\mathrm{Ag}$ & $\mathrm{Au}$ & $\mathrm{Pt}$ \\
& $m=3$, & $m=3$, & $m=3$, & $m=3$, & $m=3$, & $m=3$, \\
& $n=.5$ & $n=.5$ & $n=.5$ & $n=.2$ & $n=.2$ & $n=1$ \\
\hline$a_{0}(\AA)$ & 3.52 & 3.615 & 4.05 & 4.09 & 4.08 & 3.92 \\
& $3.52^{a}$ & $3.615^{a}$ & $4.05^{a}$ & $4.09^{a}$ & $4.08^{a}$ & $3.92^{a}$ \\
$E_{\mathrm{c}}(\mathrm{eV})$ & 4.44 & 3.49 & 3.39 & 2.85 & 3.93 & 5.77 \\
& $4.44^{a}$ & $3.49^{a}$ & $3.39^{a}$ & $2.85^{a}$ & $3.93^{a}$ & $5.77^{a}$ \\
$C_{11}$ & 2.543 & 1.695 & 0.935 & 1.215 & 1.639 & 3.167 \\
$\left(\mathrm{erg} / \mathrm{cm}^{3}\right)$ & $2.612^{b}$ & $1.700^{b}$ & $1.143^{b}$ & $1.24^{b}$ & $1.860^{b}$ & $3.467^{a}$ \\
$C_{12}$ & 1.798 & 1.227 & 0.661 & 0.916 & 1.323 & 2.661 \\
$\left(\mathrm{erg} / \mathrm{cm}^{3}\right)$ & $1.508^{b}$ & $1.225^{b}$ & $0.619^{b}$ & $0.934^{b}$ & $1.57^{b}$ & $2.507^{b}$ \\
$\mathrm{C}_{44}$ & 1.131 & 0.759 & 0.339 & 0.719 & 0.460 & 0.779 \\
$\left(\mathrm{erg} / \mathrm{cm}^{3}\right)$ & $1.317^{b}$ & $0.758^{b}$ & $0.316^{b}$ & $0.461^{b}$ & $0.42^{b}$ & $0.767^{b}$ \\
$E_{\mathrm{v}}^{\mathrm{f}}(\mathrm{eV})$ & 1.62 & 1.32 & 0.586 & 1.11 & 0.93 & 1.54 \\
\multicolumn{6}{|c}{ Potential parameters } \\
\hline$\alpha(\AA)^{-1}$ & 3.1985 & 3.19258 & 2.41395 & 3.68985 & 3.97464 & 4.75241 \\
$\beta$ & 6.21856 & 5.89702 & 6.96440 & 4.64844 & 5.23226 & 4.83662 \\
$D_{1}(\mathrm{eV})$ & 0.19915 & 0.14654 & 0.14807 & 0.12738 & 0.10667 & 0.04370 \\
$D_{2}(\mathrm{eV})$ & 2.99804 & 2.01923 & 2.97026 & 0.45715 & 0.36871 & 0.80559 \\
\hline
\end{tabular}

${ }^{a}$ from [43], ${ }^{b}$ from [44], ${ }^{c}$ from [45], ${ }^{d}$ from [46], ${ }^{e}$ from [47].

model, and by Öztekin and Colakoğlu [40] to investigate the structural phase transformation occurring in some cubic metals. The constructed model overcomes all drawbacks stated by Cai [25], and is easy for computations. The modified parts and the required other terms are as follows:

$$
\begin{aligned}
& f(r)=\exp \left[-\alpha\left(r-r_{\mathrm{e}}\right)\right], \\
& \phi\left(r_{i j}\right)=\frac{D_{1}}{(m-1)}\left[\frac{\exp \left[-m \beta\left(\frac{r}{r_{\mathrm{e}}}-1\right)\right]}{\left(\beta \frac{r}{r_{\mathrm{e}}}\right)^{n}}-\left(\beta \frac{r}{r_{\mathrm{e}}}\right)^{n} \exp \left[-\beta\left(\frac{r}{r_{\mathrm{e}}}-1\right)\right]\right], \\
& F\left(\rho_{i}\right)=-\left(E_{\mathrm{c}}-E_{\mathrm{v}}^{\mathrm{f}}\right)\left(1-\ln \sqrt{\frac{\rho_{i}}{\rho_{\mathrm{e}}}}\right) \sqrt{\frac{\rho_{i}}{\rho_{\mathrm{e}}}}+D_{2}\left(\frac{\rho_{i}}{\rho_{\mathrm{e}}}\right),
\end{aligned}
$$

where $\alpha, \beta, D_{1}$, and $D_{2}$ are fitting parameters that are determined from the lattice parameter $a_{0}$, cohesive energy $E_{\mathrm{c}}$, vacancy formation energy $\left(E_{\mathrm{v}}^{\mathbf{f}}\right)$, elastic constants $C_{i j}$, and $\rho_{\mathrm{e}}$ is the host electron density at equilibrium state and $r_{\mathrm{e}}$ is the nearest neighbour equilibrium distance. In the fitting process the cutoff distance 
is taken to be $r_{\text {cut }}=1.65 \mathrm{a}_{0}$. In this potential model there are four parameters, $\beta$ and $D_{1}$ are from a two-body term, $m$ and $n$ - adjustable selected constants. For selected values of the constants $m$ and $n$ the computed parameters and input data, for considered metals, are given in Table I. This potential can fit very well the basic properties of equilibrium state, such as, vacancy formation energy, cohesive energy, elastic constant and lattice constants.

\subsection{Stability criteria}

The Born stability criterion [1] of deformed crystal lattice on which the present calculations are based, is already presented in considerable details by Milstein [2, 3], and the usual procedure leads to the following well-known conditions:

$$
\begin{aligned}
& C_{55}>0, \quad C_{44}>0, \quad C_{22}>0, \quad C_{23}>0, \\
& C_{22}^{2}-C_{23}^{2}>0 \quad \text { and } \quad C_{11}\left(C_{22}+C_{23}\right)-2 C_{12}^{2}>0,
\end{aligned}
$$

here $C_{i j}$ are the elastic constants. Many papers exist in the literature on the stability and the related phenomena as stated in Sec. 1. Following Milstein [2, 3] and Cai [25] we can express the stresses and the complete elastic constants $C_{i j}$ in the framework of EAM by using Eq. (1) as follows:

$$
\sigma_{i}=\frac{N}{a_{j} a_{k}} \frac{\partial E}{\partial a_{i}}=\frac{N a_{i}}{2 a_{j} a_{k}}\left[F^{\prime} \sum l_{i}^{2} f^{\prime}+\frac{1}{2} \sum l_{i}^{2} \phi^{\prime}\right],
$$

where $N$ is a number of atom in a unit cell (four for fcc), $l_{i}$ are integer values $(i=1,2,3)$ for a given initial structure. $F^{\prime}=\mathrm{d} F / \mathrm{d} \rho, f^{\prime}=\mathrm{d} f / \mathrm{d} r^{2}, \phi^{\prime}=\mathrm{d} \phi / \mathrm{d} r^{2}$, and $\mathrm{d} / \mathrm{d} r^{2}=(1 / 2 r)(\mathrm{d} / \mathrm{d} r)$. The elastic moduli for a crystal under load, in terms of the second derivatives of internal energy with respect to the lattice parameters, can be written in general and explicitly for each $C_{i j}$ as

$$
\begin{aligned}
C_{i j} & =\frac{\partial^{2} E}{\partial a_{i} a_{j}}=\left(F^{\prime \prime} \sum f^{\prime} \frac{\partial r^{2}}{\partial a_{i}}\right) \sum f^{\prime} \frac{\partial r^{2}}{\partial a_{i}} \\
& +F^{\prime} \sum f^{\prime \prime} \frac{\partial r^{2}}{\partial a_{i}} \frac{\partial r^{2}}{\partial a_{j}}+F^{\prime} \sum f^{\prime} \frac{\partial^{2} r^{2}}{\partial a_{i} \partial a_{j}} \\
& +\frac{1}{2} \sum \phi^{\prime \prime} \frac{\partial r^{2}}{\partial a_{i}} \frac{\partial r^{2}}{\partial a_{j}}+\frac{1}{2} \sum \phi^{\prime} \frac{\partial^{2} r^{2}}{\partial a_{i} \partial a_{j}} \quad i, j=1, \ldots, 6, \\
C_{11} & =\frac{1}{4} a_{1}^{2} F^{\prime \prime}\left(\sum l_{1}^{2} f^{\prime}\right)\left(\sum l_{1}^{2} f^{\prime}\right)+\frac{1}{4} a_{1}^{2} F^{\prime} \sum l_{1}^{4} f^{\prime \prime} \\
& +\frac{1}{2} F^{\prime} \sum l_{1}^{2} f^{\prime}+\frac{1}{8} a_{1}^{2} \sum l_{1}^{4} \phi^{\prime \prime}+\frac{1}{4} \sum l_{1}^{2} \phi^{\prime},
\end{aligned}
$$




$$
\begin{aligned}
C_{22}= & \frac{1}{4} a_{2}^{2} F^{\prime \prime}\left(\sum l_{2}^{2} f^{\prime}\right)\left(\sum l_{2}^{2} f^{\prime}\right)+\frac{1}{4} a_{2}^{2} F^{\prime} \sum l_{2}^{4} f^{\prime \prime}+\frac{1}{2} F^{\prime} \sum l_{2}^{2} f^{\prime} \\
& +\frac{1}{8} a_{2}^{2} \sum l_{2}^{4} \phi^{\prime \prime}+\frac{1}{4} \sum l_{2}^{2} \phi^{\prime} \\
C_{12}= & \frac{1}{4} a_{1} a_{2} F^{\prime \prime}\left(\sum l_{1}^{2} f^{\prime}\right)\left(\sum l_{2}^{2} f^{\prime}\right) \\
& +\frac{1}{4} a_{1} a_{2} F^{\prime} \sum l_{1}^{2} l_{2}^{2} f^{\prime \prime}+\frac{1}{8} a_{1} a_{2} \sum l_{1}^{2} l_{2}^{2} \phi^{\prime \prime}, \\
C_{23}= & \frac{1}{4} a_{2}^{2} F^{\prime \prime}\left(\sum l_{2}^{2} f^{\prime}\right)\left(\sum l_{3}^{2} f^{\prime}\right)+\frac{1}{4} a_{2}^{2} F^{\prime} \sum l_{2}^{2} l_{3}^{2} f^{\prime \prime} \\
& +\frac{1}{8} a_{2}^{2} \sum l_{2}^{2} l_{3}^{2} \phi^{\prime \prime} \\
C_{b}= & C_{11}\left(C_{22}+C_{23}\right)-2 C_{12}^{2}, \\
C_{55}= & \frac{1}{4} a_{1} a_{2} F^{\prime}\left(\sum l_{1}^{2} l_{3}^{2} f^{\prime \prime}\right)+\frac{1}{8} a_{1} a_{2} \sum l_{1}^{2} l_{3}^{2} \phi^{\prime \prime}, \\
C_{44}= & \frac{1}{4} a_{2}^{2} F^{\prime}\left(\sum l_{2}^{2} l_{3}^{2} f^{\prime \prime}\right)+\frac{1}{8} a_{2}^{2} \sum l_{2}^{2} l_{3}^{2} \phi^{\prime \prime}, \\
C_{2} & \\
C_{2} &
\end{aligned}
$$

where $F^{\prime \prime}=\mathrm{d}^{2} F / \mathrm{d} \rho^{2}, f^{\prime \prime}=\mathrm{d}^{2} f /\left(\mathrm{d} r^{2}\right)^{2}, \phi^{\prime \prime}=\mathrm{d}^{2} \phi / \mathrm{d}\left(r^{2}\right)^{2}$.

To use the above expressions in numerical manner for the present purpose, the lattice parameter $a_{1}$ is increased by a small amount of $\Delta a_{1}$ and then the lattice parameters $a_{2}=a_{3}$ are computed by permitting their values to the state for which $\sigma_{2}=\sigma_{3}=0$, where $\sigma_{2}$ and $\sigma_{3}$ are evaluated from Eq. (6); then $a_{1}$ is held constant while $a_{2}$ and $a_{3}$ are allowed to relax in this manner. By using these values of the lattice parameters, we calculate the applied stress $\sigma_{1}$ and the required elastic constants $C_{i j}$. The obtained elastic constants are applied to the stability conditions (5). This process is repeated many times to examine the curve of stress versus lattice parameter $a_{1}$ and the stability of lattice throughout the desired range.

\subsection{Third-order elastic constants}

The present embedded energy function is also used to evaluate the third-order elastic constants (TOEC) $C_{111}, C_{112}, C_{123}, C_{144}, C_{166}$, and $C_{456}$ for the testing its validity. The general forms for higher-order elastic constants are found in the 
early paper of Brugger [41]. Recently Chantasirivan and Milstein [42] have developed a certain explicit expression for TOEC in the framework of the EAM, and the general form of them is as follows:

$$
\begin{aligned}
C_{I J K} & =\frac{1}{\Omega_{0}}\left\{F^{\prime \prime \prime}\left(\sum \frac{X_{I} f^{\prime}}{r}\right)\left(\sum \frac{X_{J} f^{\prime}}{r}\right)\left(\frac{X_{K} f^{\prime}}{r}\right)+F^{\prime \prime}\left(\sum \frac{X_{I} f^{\prime}}{r}\right)\right. \\
\times & {\left[\sum \frac{X_{J} X_{K}}{r^{2}}\left(f^{\prime \prime}-\frac{f^{\prime}}{r}\right)\right]+F^{\prime \prime}\left(\sum \frac{X_{J} f^{\prime}}{r}\right)\left[\sum \frac{X_{I} X_{K}}{r^{2}}\left(f^{\prime \prime}-\frac{f^{\prime}}{r}\right)\right] } \\
+ & F^{\prime \prime}\left(\sum \frac{X_{K} f^{\prime}}{r}\right)\left[\sum \frac{X_{I} X_{J}}{r^{2}}\left(f^{\prime \prime}-\frac{f^{\prime}}{r}\right)\right] \\
+ & F^{\prime} \sum \frac{X_{I} X_{J} X_{K}}{r^{3}}\left(f^{\prime \prime}-\frac{3 f^{\prime \prime}}{r}+3 \frac{f^{\prime}}{r^{2}}\right) \\
+ & \left.\frac{1}{2} \sum \frac{X_{I} X_{J} X_{K}}{r^{3}}\left(\phi^{\prime \prime \prime}-3 \frac{\phi^{\prime \prime}}{r}+3 \frac{\phi^{\prime}}{r^{2}}\right)\right\}
\end{aligned}
$$

where $X_{I}=x_{i} x_{j}$ and $I$ is the Voight contraction of $i j$. The primes on the $F$ and $f$ functions represent the first, second, and third order derivatives with respect to $\rho$ and $r$. By using this formalism we calculate the TOEC and the obtained results are listed in Table IV.

\subsection{Pressure-volume $(P-V)$ calculations}

When a cubic crystal with pairwise central-force interactions between the atoms is subjected to an external force applied normal to the face of the unit cell, the internal force upon the face of the unit cell is given [3] by

$$
F_{i}=\frac{1}{4} N a_{i} \sum_{l_{1}, l_{2}, l_{3}} l_{i}^{2} \frac{\partial \phi}{\partial r^{2}}
$$

with

$$
r=\frac{1}{2}\left(a_{1}^{2} l_{1}^{2}+a_{2}^{2} l_{2}^{2}+a_{3}^{2} l_{3}^{2}\right)^{1 / 2}
$$

The direction of the force is along $a_{i}(i=1,2$, or 3$)$ and $a_{i}$ is the cell length. Under hydrostatic pressure all $a_{i}$ are equal and the pressure $P$ becomes

$$
P=\frac{F_{i}}{a^{2}}=\frac{N}{4 a} \sum_{l_{1}, l_{2}, l_{3}} l_{i}^{2} \frac{\partial \phi}{\partial r^{2}}
$$

and the corresponding volume is

$$
V / V_{0}=\left(a / a_{0}\right)^{3} .
$$

If we transform the notations in (17) into the present framework of EAM, the pressure $P$ can be written as 


$$
P=\frac{N}{2 a}\left(F^{\prime} \sum l_{i}^{2} \frac{\partial f}{\partial r^{2}}+\frac{1}{2} \sum l_{i}^{2} \frac{\partial \phi}{\partial r^{2}}\right)
$$

where $F^{\prime}$ is as stated above.

Pressure-volume relations are investigated on this line by Milstein [3] for $\mathrm{Ni}$, and Thakur [27] for $\mathrm{Ni}$, Th, Mo, and $W-\alpha$ by using logarithmic or Morse type potentials. More recently, Chantasirivan and Milstein [18] are also reproduced the $\mathrm{P}-\mathrm{V}$ curves based on Rose's universal equation of state (EOS) for $\mathrm{Al}, \mathrm{Na}, \mathrm{Cu}$, and Mo by making use of the EAM model. Our present results for $\mathrm{P}-\mathrm{V}$ behaviour of the studied metals $\mathrm{Al}, \mathrm{Cu}, \mathrm{Ni}$, and $\mathrm{Ag}$ are shown in Fig. 4 with experimental points from Ref. [52].

\section{Results and discussion}

On the structural phase transformation and elastic instability, the numerical calculations are performed using the EAM model for fcc metals $\mathrm{Ni}, \mathrm{Cu}, \mathrm{Ag}$, $\mathrm{Al}, \mathrm{Au}$, and Pt under [100] uniaxial loading. Third-order elastic constants and pressure-volume behaviour are also included in the computations. In our EAM model, the energy and its first two derivatives are continuous so that their values at the cut-off distance $r_{c}$ are, almost, zero when a long range force is considered. As can be seen from Table $\mathbf{I}$, the fitting results, the calculated lattice constants

TABLE II

Theoretical lattice parameters $(a)$, atomic volumes $(V)$ and cohesive energies $(E)$ per atom of the unstressed bcc and fcc structures for $\mathrm{Ni}, \mathrm{Cu}, \mathrm{Ag}, \mathrm{Al}, \mathrm{Au}$, and Pt.

\begin{tabular}{c|c|c|c|c|c|c|c}
\hline \hline Met. & $a_{\mathrm{bcc}}(\AA)$ & $V_{\mathrm{bcc}}(\AA)^{3}$ & $\frac{E_{\mathrm{bcc}}}{\left(10^{-12} \mathrm{erg}\right)}$ & $a_{\mathrm{fcc}}(\AA)$ & $V_{\mathrm{fcc}}(\AA)^{3}$ & $\frac{E_{\mathrm{fcc}}}{\left(10^{-12} \operatorname{erg}\right)}$ & $\frac{E_{\mathrm{fcc}}-E_{\mathrm{bcc}}}{\left(10^{-12} \mathrm{erg}\right)}$ \\
\hline $\mathrm{Ni}$ & 2.8095 & 11.0880 & -6.9652 & 3.5200 & 10.9035 & -7.0669 & -0.1017 \\
& & & & $3.52^{a}$ & & $-7.104^{a}$ & $-0.1696[48]$ \\
$\mathrm{Cu}$ & \multirow{2}{*}{2.8844} & \multirow{2}{*}{11.9988} & -5.5094 & 3.616 & 11.8202 & -5.5832 & -0.0738 \\
& & & & $3.615^{a}$ & & $-5.584^{a}$ & - \\
$\mathrm{Al}$ & \multirow{2}{*}{3.2410} & \multirow{2}{*}{17.022} & -5.0504 & 4.05 & 16.6075 & -5.1173 & -0.0669 \\
& & & & $4.05^{a}$ & & $-5.424^{a}$ & $-0.1120[49]$ \\
$\mathrm{Ag}$ & \multirow{3}{*}{3.2600} & \multirow{2}{*}{17.3229} & -4.4784 & 4.09 & 17.1044 & -4.5387 & -0.0603 \\
& & & & $4.09^{a}$ & & $-4.561^{a}$ & $-0.2080[48]$ \\
$\mathrm{Au}$ & \multirow{2}{*}{3.2500} & \multirow{2}{*}{17.1641} & -6.2216 & 4.078 & 16.9543 & -6.2847 & -0.0631 \\
& & & & $4.08^{a}$ & & $-6.288^{a}$ & $-0.1856[48]$ \\
& \multirow{2}{*}{3.1400} & \multirow{2}{*}{15.4795} & -9.1437 & 3.93 & 15.1862 & -9.2304 & -0.0867 \\
& & & & $3.92^{a}$ & & $-9.232^{a}$ & $-0.3840[48]$ \\
\hline
\end{tabular}

${ }^{a}$ experiment [17], ${ }^{b}$ first principles calculations [48, 49], $V=c(a / 2)^{3}, c$ is 2 and 4 for fcc and bcc structures, respectively, and $a$ is the lattice parameters. 
and cohesive energy are in excellent agreement with experimental data. The calculated elastic constants are, moderately, in agreement with experiment. The most of numerical results obtained from this work are summarized in Figs. 1, 2, 3, 4 and Tables II, III, IV.

TABLE III

Values of cell lengths $a_{1}, a_{2}\left(=a_{3}\right)$ in tension and in compression for $\mathrm{Ni}, \mathrm{Cu}, \mathrm{Al}, \mathrm{Ag}$, $\mathrm{Au}$, and $\mathrm{Pt}$ for the stress $\sigma_{1}$ (theoretical strength) where the Born stability criteria are violated.

\begin{tabular}{|c|c|c|c|c|c|c|c|c|}
\hline \multirow{3}{*}{ Met. } & \multirow{2}{*}{\multicolumn{3}{|c|}{$\begin{array}{c}\text { MSR-I } \\
\text { Failure in tens. } C_{\mathrm{a}}\end{array}$}} & \multirow{2}{*}{\multicolumn{3}{|c|}{$\begin{array}{l}\text { MSR-I } \\
\text { Failure in compr. } C_{\mathrm{b}}\end{array}$}} & \multicolumn{2}{|c|}{ MSR-II } \\
\hline & & & & & & & \multirow{2}{*}{$\frac{C_{23}}{a_{1}(\AA)}$} & \multirow{2}{*}{$\frac{C_{b}}{a_{1}(\AA)}$} \\
\hline & $a_{1}(\AA)$ & $a_{2}(\AA)$ & $\sigma_{1}(\mathrm{GPa})$ & $a_{1}(\AA)$ & $a_{2}(\AA)$ & $\sigma_{1}(\mathrm{GPa})$ & & \\
\hline \multirow[t]{2}{*}{$\mathrm{Ni}$} & 3.858 & 3.422 & 7.809 & 3.200 & 3.691 & -3.249 & 2.108 & 2.709 \\
\hline & & & $6.10^{a}$ & & & & & \\
\hline \multirow[t]{3}{*}{$\mathrm{Cu}$} & 3.580 & 3.514 & 5.279 & 2.288 & 3.792 & -2.132 & 2.176 & 2.797 \\
\hline & & & $3.0^{b}$ & & & & & \\
\hline & & & $6.97^{c}$ & & & & & \\
\hline \multirow[t]{2}{*}{$\mathrm{Al}$} & 4.461 & 3.941 & 3.138 & 3.682 & 4.253 & -1.432 & 1.990 & 3.057 \\
\hline & & & $2.254^{d}$ & & & & & \\
\hline \multirow[t]{2}{*}{$\mathrm{Ag}$} & 4.476 & 3.961 & 2.849 & 3.713 & 4.293 & -1.111 & 2.451 & 3.173 \\
\hline & & & $1.89^{e}$ & & & & & \\
\hline $\mathrm{Au}$ & 4.466 & 3.944 & 3.137 & 3.703 & 4.282 & -1.282 & 2.370 & 3.129 \\
\hline \multirow[t]{2}{*}{$\mathrm{Pt}$} & 4.295 & 3.802 & 4.429 & 3.570 & 4.133 & -1.733 & 2.349 & 3.051 \\
\hline & & & $6.71^{e}$ & & & & & \\
\hline
\end{tabular}

${ }^{a}$ from [48], ${ }^{b}$ from [49], ${ }^{c}$ from [16], ${ }^{d}$ from [50], ${ }^{e}$ from [10].

Energy versus lattice parameters curves are plotted in Fig. 1 for each metal considered. In each curve there are two special points marked with circle and square, represent the minimum of energy, and the energy at the inflection point through which the second-order derivative of energy with respect to $a_{1}$ changes sign, respectively. Our numerical results show that the points marked with circle assert the initial equilibrium state of the fcc structures and the points marked with triangles occupy the stress-free face-centred tetragonal (fct) structures. The squares indicate the stress-free bcc phases, where the ratio $a_{2}$ to $a_{1}$ (with $a_{2}=a_{3}$ ) is taken to be $\sqrt{2}$. In a usual treatment a bcc crystal is handled as a fct crystal. We can see, from Fig. 1, that the predicted bcc phase is unstable for all metals considered here.

The stresses $\sigma_{1}$ versus cell length $a_{1}$ are shown in Fig. 2. The circle and square marks on the curve are associated with the stress-free cell length $a_{\mathrm{bcc}}$ and $a_{\mathrm{fcc}}$. These are calculated from the values of marked points, respectively. It can be seen from Table II that the theoretical lattice parameter $a_{\mathrm{fcc}}$ and energy $E_{\mathrm{fcc}}$ 
TABLE IV

Third-order elastic constants $\left(C_{i j k}\right)$ in EAM for some fCc metals (in unit $10^{12} \mathrm{dyn} / \mathrm{cm}^{2}$ ).

\begin{tabular}{c|c|c|c|c}
\hline \hline$C_{i j k}$ & $\mathrm{Al}$ & $\mathrm{Ni}$ & $\mathrm{Cu}$ & $\mathrm{Ag}$ \\
& $(m=2.0$, & $(m=2.0$, & $(m=2.0$, & $(m=2.0$ \\
& $n=0.0)$ & $n=0.1)$ & $n=0.1)$ & $n=0.2)$ \\
& $(m=3.0$, & $(m=2.0$, & $(m=3.0$, & $(m=2.5$ \\
& $n=0.2)$ & $n=0.0)$ & $n=0.2)$ & $n=0.2)$ \\
\hline This work & -7.677 & -11.931 & -11.552 & 8.664 \\
$C_{111}$ & -6.945 & -21.938 & -14.102 & -10.515 \\
Exp [51] & -5.390 & -14.370 & -10.400 & -8.170 \\
\hline This work & -4.194 & -8.255 & -7.001 & -5.039 \\
$C_{112}$ & -3.851 & -12.525 & -8.066 & -5.807 \\
Exp [51] & -4.060 & -10.530 & -7.700 & -5.870 \\
\hline This work & 0.539 & 0.594 & 0.034 & 0.363 \\
$C_{123}$ & 0.245 & 0.843 & 0.291 & 0.852 \\
Exp [51] & 0.530 & 1.190 & 0.920 & 0.540 \\
\hline This work & -0.281 & -0.126 & -0.124 & -2.201 \\
$C_{144}$ & -0.241 & -0.519 & -0.229 & -0.446 \\
Exp [51] & 0.230 & -1.400 & -0.030 & 0.560 \\
\hline This work & -3.670 & -8.065 & -6.529 & -4.616 \\
$C_{166}$ & -3.475 & -11.499 & -7.487 & -5.159 \\
Exp [51] & -3.400 & -9.200 & -7.800 & -6.370 \\
\hline This work & -0.215 & -0.741 & -0.573 & -0.294 \\
$C_{456}$ & -0.222 & -0.730 & -0.523 & -0.278 \\
Exp [51] & -0.300 & -0.700 & -0.950 & 0.830 \\
& & & &
\end{tabular}
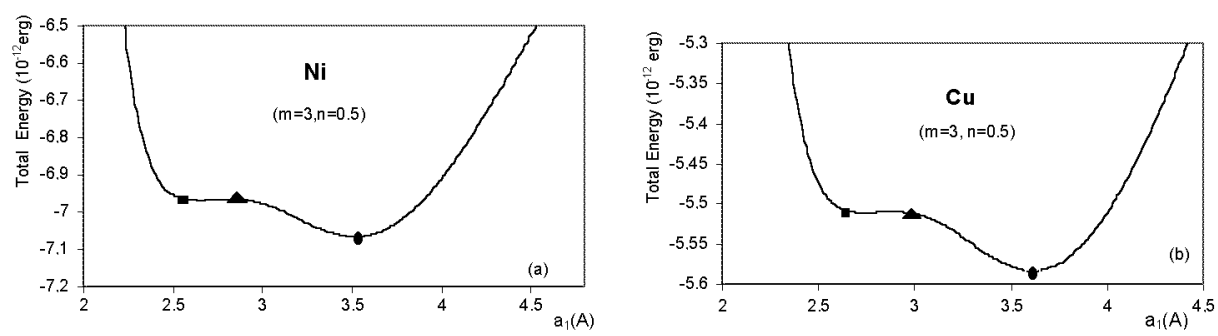

Fig. 1. Dependence of cohesive energy per atom on $a_{1}$ for (a) $\mathrm{Ni}$ and (b) Cu.

of fcc structures are in good agreement with experimental values. From the same table one can also see that our calculated fcc-bcc energy differences are very close to the obtained results from the first-principle calculations [32]. 

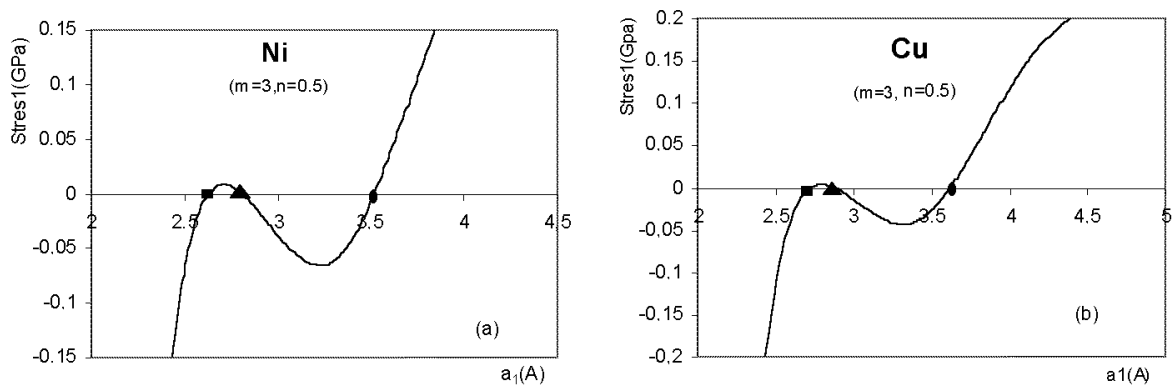

Fig. 2. Theoretical values of applied stress $\sigma_{1}$ calculated as a function of $a_{1}$ for (a) $\mathrm{Ni}$ and (b) $\mathrm{Cu}$.


Fig. 3. The variations of $C_{55}, C_{44}, C_{22}, C_{a}$, and $C_{b}$ (in arb. units) as a function of $a_{1}$ for (a) Ni, and (b) Cu.

In order to see whether the bcc phase is stable or not, we plot $C_{55}, C_{44}, C_{22}$, $C_{a}\left(=C_{22}^{2}-C_{23}^{2}\right)$ and $C_{b}\left(=C_{11}\left(C_{22}+C_{23}\right)-2 C_{12}^{2}\right)$ as a function of cell length $a_{1}$ in Fig. 3. Figure 3 shows, for $\mathrm{Ni}$, that in tension, when the cell length $a_{1}$ is smaller than $3.858 \AA, C_{a}$ becomes negative, and in compression when $a_{1}$ larger than $3.2 \AA$, 

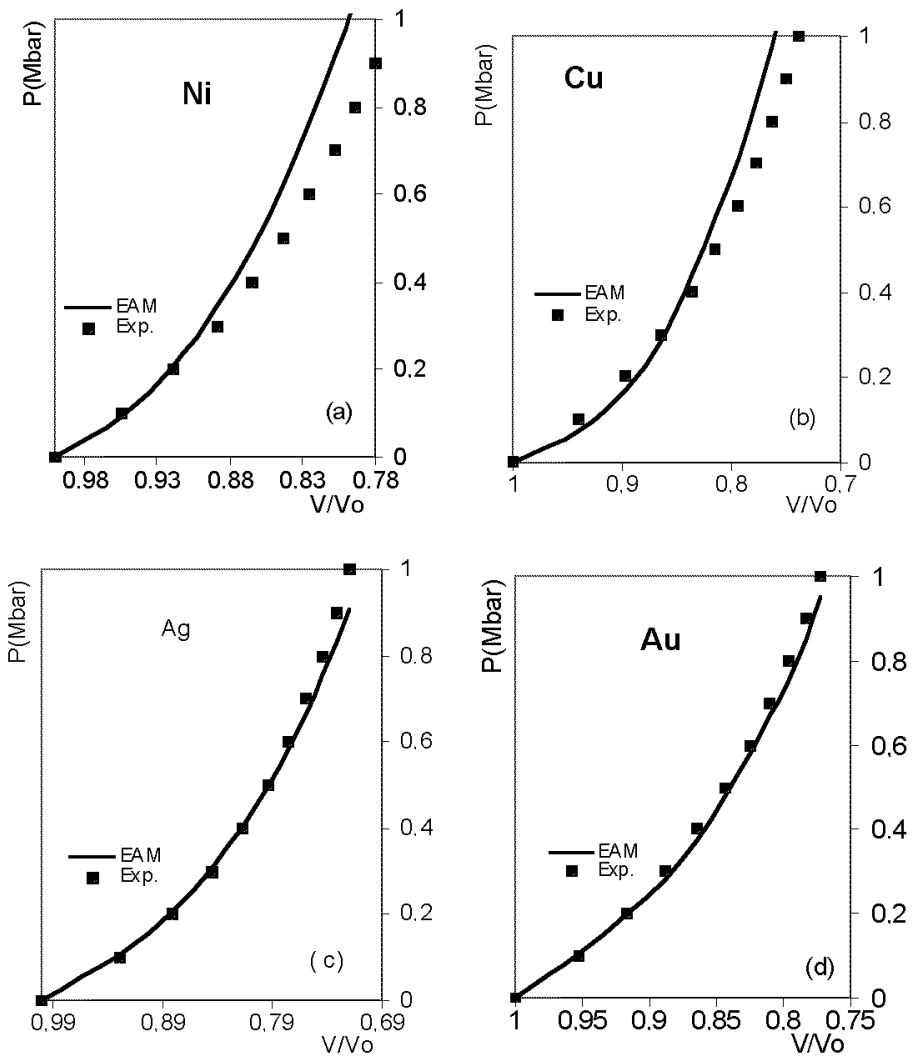

Fig. 4. $P$ versus $V / V_{0}$ behaviour for (a) Ni, (b) $\mathrm{Cu}$, (c) Ag, and (d) Au.

$C_{b}$ is first violated. Thus, according to the criteria given in Eq. (5), Ni under [100] loading has an elastic stability region (ESR) and corresponds to the internal of the variation of lattice parameter $a_{1}$ between $3.2 \AA$ and $3.858 \AA$. These ranges are given for other metals considered in Table III. From Fig. 2 we can also find that only the fcc phase is predicted to fall into the mechanic stability region (MSR). Hence, from the point of view of mechanical stability, fcc phase is stable, but bcc phase is unstable. From Fig. 3 it is seen that the failure first occurs in tension when $C_{b}>0$ is violated and in compression when $C_{b}>0$ is violated. Table III summarizes the values of lattice parameter $a_{1}$ and stress (theoretical strength) at failure in tension and in compression. Table III shows MSR-I and MSR-II for all fcc metals considered. The region of lattice stability determined from the failure criteria shown in Fig. 3, as can be seen in Fig. 2, the theoretical strengths in tension and compression are taken to be the stresses observed in Fig. 2 at the boundaries of the region stability. Table III lists the values of stress $\sigma_{1}$ calculated at failure in tension and in compression. Experimental and/or other theoretical strength values are also given in Table III for the sake of compression. 
The third-order elastic constants and $\mathrm{P}-\mathrm{V}$ behaviour are also computed. The obtained agreement between theory and experimental values supports the validity of the present model. The evaluated values together with experimental results are listed in Table IV. Finally, Fig. 4 indicates the $\mathrm{P}-\mathrm{V}$ relations for the studied metals. The agreement is, generally, good in low pressure, but at higher pressure values the deviations become significant.

The present pair potential in EAM is, for the first time used for this purpose and in this model, there are not too many potential parameters. As a result, we present a simple and completely analytical modified EAM potential model. The potential can be used to correctly describe the structural phase transformation, elastic instability, pressure versus volume relations and third-order elastic constants for fec metals.

\section{Acknowledgments}

The authors are thankful to Dr. J. Cai for providing his parameterizing code.

\section{References}

[1] M. Born, Proc. Cambridge Phil. Soc. (U.K.) 36, 160 (1940).

[2] F. Milstein, Phys. Rev. B 3, 1130 (1971).

[3] F. Milstein, J. Appl. Phys. 44, 3825 (1973).

[4] F. Milstein, R. Hill, K. Huang, Phys. Rev. B 21, 4282 (1980).

[5] A.K. Mitra, P.K. Sengupta, J. Phys. F, Met. Phys. 13, 2221 (1983).

[6] J. Marshall, F. Milstein, J. Phys. F, Met. Phys. 18, 1913 (1988).

[7] F. Milstein, J. Marschall, Acta Metall. Mater. 40, 1229 (1992).

[8] F. Milstein, H.E. Fang, J. Marschall, Philos. Mag. A 70, 621 (1994).

[9] M. Senoo, I. Fujishiro, M. Hirano, Bull. JSME 27, 2680 (1984).

[10] K.B. Seyoum, K.P. Thakur, D. Jha, Phys. Status Solidi B 167, 495 (1991) and references therein.

[11] K. Huang, F. Milstein, J.A. Boldwin, Phys. Rev. B 10, 3635 (1974).

[12] F. Milstein, R. Hill, J. Mech. Phys. Solids 25, 457 (1977); 26, 213 (1978); 27, 255 (1979).

[13] N.H. Macmillan, A. Kelly, Proc. R. Soc. Lond. A 330, 309 (1972).

[14] N.H. Macmillan, A. Kelly, Mater. Sci. Eng. 12, 79 (1973).

[15] F. Milstein, K. Huang, Phys. Rev. B 18, 2529 (1978).

[16] F. Milstein, B. Farber, Phys. Rev. Lett. 44, 277 (1980); F. Milstein, J. Marschall, H.E. Fang, Phys. Rev. Lett. 74, 2977 (1995).

[17] T. Suzuki, H.M. Ledbetter, Philos. Mag. A 48, 83 (1983).

[18] F. Milstein, S. Chantasiriwan, Phys. Rev. B 58, 6006 (1998); ibid. 58, 5996 (1998).

[19] T. Kraft, P.M. Marcus, Phys. Rev. B 48, 5886 (1993). 
[20] G. Singh, Phys. Status Solidi B 164, 401 (1991).

[21] P. Alippi, P.M. Marcus, M. Scheffler, Phys. Rev. Lett. 78, 3892 (1997).

[22] F. Milstein, D.J. Rasky, Phys. Rev. B 54, 7036 (1996).

[23] K.P. Thakur, S. Ahmed, Phys. Status Solidi B 144, 529 (1987); ibid. 155, 445 (1989); Phys. Rev. B 35, 607 (1987).

[24] N.H. Macmillan, J. Mater. Sci. 7, 239 (1972).

[25] J. Cai, Phys. Status Solidi B 203, 345 (1997).

[26] J. Koike, Phys. Rev. B 47, 7700 (1993).

[27] K.P. Thakur, J. Phys. F, Met. Phys. 15, 2421 (1985).

[28] R. Najafabadi, G. Kalonji, Acta Metall. 36, 917 (1988).

[29] F. Milstein, D.J. Rasky, Phys. Rev. B 33, 2341 (1986).

[30] G. Singh, Phys. Status Solidi B 161, 145 (1990).

[31] R.M. Wentzcovitch, H. Krakauer, Phys. Rev. B 42, 4563 (1990).

[32] M. Sigalas, D.A. Papaconstontopoulos, N.C. Bacalis, Phys. Rev. B 45, 5777 (1992)

[33] M.J. Mehl, L.L. Boyer, Phys. Rev. B 43, 9498 (1991).

[34] Q. Xie, M.C. Huang, Phys. Status Solidi B 186, 393 (1994).

[35] M.S. Daw, M.I. Baskes, Phys. Rev. B 29, 6443 (1984).

[36] M.S. Daw, S.M. Foiles, M.I. Baskes, Mater. Sci. Rep. 9, 251 (1993).

[37] Z. Yifang, Z. Bengwei, L. Shuzhi, J. Zhanpeng, J. Phys. B 101, 161 (1996).

[38] A.E. Carlsson, in: Solid State Physics: Advanced in Research and Applications, Eds. H. Ehrenreich, D. Turnbull, Vol. 43, Academic, Boston 1990, p. 1.

[39] M.L. Verma, R.P.S. Rathore, Phys. Status Solidi B 185, 93 (1994).

[40] Y. Öztekin, K. Colakoğlu, Turkish J. Phys. 21, 930 (1997).

[41] K. Brugger, Phys. Rev. A 133, 1611 (1964).

[42] S. Chantasiriwan, F. Milstein, Phys. Rev. B 48, 14080 (1996).

[43] C. Kittel, Introduction to Solid State Physics, 5th ed., Wiley, New York 1976.

[44] N.H. Macmillan, A. Kelly, Mater. Sci. Eng. 12, 79 (1973).

[45] W. Wycisk, M. Feller-Knipmeimer, J. Nucl. Mater 69, 616 (1978).

[46] R.W. Balluffi, J. Nucl. Mater. 69-70, 240 (1978).

[47] J.S. Koehler, in: Vacancies and Interstitials in Metals, Eds. A. Seeger, D. Schumacher, W. Schilling, J. Diehl, North-Holland, Amsterdam 1970, p. 169.

[48] M.J. Mehl, L.J. Boyer, Phys. Rev. B 43, 9498 (1991).

[49] A. Voter, S.P. Chen, in: Characterization of Defects in Materials, MRS Symp. Proc., No. 82, Eds. R.V. Siegal, J.R. Weemorn, R. Sinclair, MRS, Pittsburgh 1987, p. 175.

[50] S.S. Brenner, J. Appl. Phys. 27, 1484 (1956).

[51] Y. Hiki, A.V. Granato, Phys. Rev. 44, 411 (1966).

[52] R.G. McQueen, S.P. Marsh, J. Appl. Phys. 31, 1253 (1960). 